\title{
Self-organized Criticality and Standard Model Parameters
}

\author{
Ervin Goldfain \\ Research Scholar, Ronin Institute, Montclair, New Jersey 07043 \\ Email: ervin.goldfain@ronininstitute.org
}

\begin{abstract}
As paradigm of complex behavior, Self-organized Criticality (SOC) reflects the ability of nonequilibrium dynamical systems to self-adjust into metastable states that are scale independent. The goal of this report is to tentatively show that the hierarchy of Standard Model masses and mixing angles follows from the universal scaling attributes of SOC.
\end{abstract}

Key words: Standard Model, fermion mass hierarchy, mixing matrices, nonlinear dynamics and chaos, bifurcations, Feigenbaum's universality.

\section{Introduction}

The generation structure of quarks and leptons stands out as one of the most intriguing puzzles of theoretical high-energy physics [1]. The conventional formulation of the SM requires 19 free input parameters, among which 12 can be expressed in terms of empirical mass eigenvalues. In addition, there is a set of four inputs determined by the so-called Cabibbo-Kobayashi-Maskawa (CKM) matrix whose structure includes three quarkmixing angles and one $\mathrm{CP}$ phase. The remaining three parameters are two gauge couplings $\left(\alpha_{E M}, \alpha_{Q C D}\right)$ and the strong CP phase. Experiments have convincingly confirmed the existence of neutrino oscillations and masses, yet our understanding of the neutrino sector is currently incomplete. There is a large body of proposed extensions of SM, each of them aiming to resolve some unsatisfactory aspects of the theory while introducing new unknowns. It is our view that, at least at the time of writing, these attempts fail to offer arguments with clear relevance for the physics of SM. In contrast, our work suggests that the pattern of fermion masses and mixing angles is rooted in the bifurcation regime of nonlinear dynamics, a scenario backed up by numerous experiments carried out across various disciplines.

The paper is structured in the following way: next section details the main working assumptions on which the paper is founded, section three introduces self-organized criticality (SOC) and its connection to the minimal fractal manifold (MFM) geometry of spacetime near the Fermi scale. Section four delves into the scaling hierarchies of fermions in relation to universal bifurcations and the Cabibbo angle.

\section{Assumptions}

Our work develops from three underlying premises, namely,

A1) As complex dynamics is likely to come into play near or above the Fermi scale, SOC becomes a suitable framework for modeling the behavior of field theory beyond $\mathrm{SM}[2-3,15]$ 
A2) MFM and its theoretical implications, in particular, the running of spacetime dimensions with the energy scale, assumes a leading role [3].

A3) in line with A1), our derivation relies on the center manifold theory and the reduction of a generic large system of ODE's to a quadratic equation [4-5]. A direct consequence of this conjecture is that the transition to chaos in the dynamics of field theory follows the Feigenbaum's route of period-doubling bifurcations. [6-10]

\section{From SOC to the minimal fractal manifold (MFM)}

The study of equilibrium critical phenomena reveals that, near a second-order phase transition, the scaling behavior of physical observables follows the so-called finite-size scaling (FSS) ansatz. By analogy, the probability distribution defining the FSS ansatz in SOC takes the form [2]

$$
\begin{gathered}
P(s, L) \sim s^{-\tau_{s}} \Phi\left(s / s_{c}\right) \text { for } s>1, L>>1 \\
s_{c}(L) \sim L^{D_{s}} \text { for } L>>1
\end{gathered}
$$

in which $s_{c}$ is the cutoff in the avalanche-size and where $\tau_{s}$ and $D_{S}$ are called the avalanche-size exponent and the avalanche dimension, respectively. The cutoff function $\Phi(x)$ assumes the following expansion around zero,

$$
\Phi(x) \sim\left\{\begin{aligned}
\Phi(0)+\Phi^{\prime}(0) & x+\frac{1}{2} \Phi^{\prime \prime}(0) x^{2}+\ldots, & & x<1 \\
& \rightarrow 0, & & x>1
\end{aligned}\right.
$$

The transition from the framework of equilibrium critical phenomena to SOC can be made under the plausible assumption that the correlation length $\xi$ displays the same behavior as the avalanche-size, i.e.,

$$
s=\xi ; \quad s_{c}=L
$$

MFM denotes a spacetime continuum characterized by arbitrarily small and scaledependent deviations from four dimensions $\left(\varepsilon=4-D=O\left(\mathrm{~m}^{2} / \Lambda_{U V}^{2}\right)<<1\right)$ [3]. It reflects an evolving setting that starts far-from-equilibrium and gradually reaches the equilibrium conditions mandated by relativistic field theory in the limit of four-dimensional spacetime $(\varepsilon=0)$.

Elaborating from this baseline and appealing to A1) and A2), we advance the hypothesis that the dimensional deviation $\varepsilon$ and the avalanche-size $s=\xi$ are interchangeable concepts via

$$
\varepsilon=4-D=s^{-1}<<1
$$

This ansatz is consistent with the spirit of conformal field theory, as the four-dimensional limit $\varepsilon=0,(D=4)$ naturally matches the asymptotic approach to the far-infrared regime 
of massless fields $\left(m=\xi^{-1}=s^{-1} \rightarrow 0\right)$. Further setting the dimensional cutoff in close proximity to zero,

$$
\varepsilon_{c}=\varepsilon_{\infty}<<\varepsilon<<1
$$

turns the probability distribution (1) into

$$
\begin{aligned}
& P\left(\varepsilon, \varepsilon_{c}\right) \sim \varepsilon^{\tau_{s}} \Phi\left(\varepsilon_{\infty} / \varepsilon\right), \varepsilon<<1 \\
& \varepsilon_{\infty}(\mu) \sim \mu^{D_{s}}, \mu>>1, D_{s}<0
\end{aligned}
$$

where $\mu$ is the dimensionless Renormalization Group scale and $\tau_{s}=2$ for quantum mechanical paths and random-walk models in three dimensional space.

Assumption A3) implies that the dimensional flow of $\varepsilon$ towards $\varepsilon_{\infty}$ obeys the power-law

$$
\varepsilon_{n}-\varepsilon_{\infty}=O\left(\varepsilon_{n}\right) \sim \bar{\delta}^{-n}
$$

where $\bar{\delta}$ stands for a Feigenbaum-like constant and $n$ for the flow iteration number. By (2), (5)-(7), we arrive at the expansion

$$
P\left(\varepsilon_{n}, \varepsilon_{\infty}\right) \sim \Phi(0) \bar{\delta}^{-2 n}+\Phi^{\prime}(0) \varepsilon_{\infty} \bar{\delta}^{-n}+\ldots
$$

The second term of (8) is non-vanishing if $\Phi^{\prime}(0)$ assumes singular values according to

$$
\Phi^{\prime}(0)=O\left(\varepsilon_{\infty}^{-1}\right)>>1
$$

Also note that (8) stays finite only if terms containing second and higher-order derivatives of the cutoff function are properly suppressed by a regularization procedure or by fluctuations that cancel out. If these conditions are fulfilled, the probability distribution (8) boils down to only a couple of terms depending on the iteration number, the constant $\bar{\delta}$ and its square. We are now ready to explore the link the between (8) and the scaling distribution of fermion masses and mixing angles.

\section{Scaling hierarchies of the fermion sector}

A remarkable yet unexplained property of SM parameters is that they are organized in a hierarchical pattern. The scaling ratio of two parameters in the hierarchy depends on integer powers of the Cabibbo angle whose experimental best-fit value is $\theta_{C}=12.9-13^{\circ}$. It is customary to work with the Cabibbo angle in the equivalent trigonometric form, that is, $\lambda=\sin \theta_{C}=0.223-0.225$. Let us denote the set of charged lepton and current-quark masses, evaluated at an arbitrary energy scale, by the vector $M_{l}$ and matrix $M_{q}$, respectively, 


$$
M_{l}=\left[\begin{array}{lll}
m_{e} & m_{\mu} & m_{\tau}
\end{array}\right] \quad M_{q}=\left[\begin{array}{ll}
m_{u} & m_{d} \\
m_{c} & m_{s} \\
m_{t} & m_{b}
\end{array}\right]
$$

The explicit set of mass scaling ratios built from (10) is given by [1]

$$
\begin{array}{ll}
\frac{m_{e}}{m_{\mu}}=O\left(\lambda^{4}\right) & \frac{m_{\mu}}{m_{\tau}}=O\left(\lambda^{2}\right) \\
\frac{m_{c}}{m_{t}}=O\left(\lambda^{4}\right) & \frac{m_{s}}{m_{b}}=O\left(\lambda^{2}\right) \\
\frac{m_{u}}{m_{t}}=O\left(\lambda^{8}\right) & \frac{m_{d}}{m_{b}}=O\left(\lambda^{4}\right)
\end{array}
$$

The CKM matrix expressed using the Wolfenstein parameterization is approximated to the leading order by entries dependent on powers of $\lambda$, namely,

$$
V_{C K M}=\left|\begin{array}{ccc}
1 & \lambda & \lambda^{3} \\
-\lambda & 1 & \lambda^{2} \\
\lambda^{3} & -\lambda^{2} & 1
\end{array}\right|
$$

A similar matrix composition may be assigned to mixing in the lepton sector. If the neutrino mass matrix $m_{v}$ and the charged lepton matrix $m_{l}$ are diagonalized through the following transformations [1]

$$
\begin{aligned}
& m_{v}=U_{v} m_{v}^{\text {diag }} U_{v}^{T} \\
& m_{l}=U_{L} m_{l}^{\text {diag }} U_{R}^{+}
\end{aligned}
$$

then it can be shown that the neutrino mixing matrix (PMNS matrix) may be formulated as

$$
U_{P M N S}=U_{L}^{+} U_{v}
$$

which leads to the following representation

$$
m_{l} m_{l}^{+} \sim m_{\tau}^{2}\left|\begin{array}{ccc}
\lambda^{6} & \lambda^{5} & \lambda^{3} \\
\lambda^{5} & \lambda^{4} & \lambda^{2} \\
\lambda^{3} & \lambda^{2} & 1
\end{array}\right|
$$




$$
U_{L} m_{l}^{\text {diag }} U_{L}^{T} \sim m_{\tau}\left|\begin{array}{ccc}
\lambda^{4} & \lambda^{3} & \lambda^{3} \\
\lambda^{3} & \lambda^{2} & \lambda^{2} \\
\lambda^{3} & \lambda^{2} & 1
\end{array}\right|
$$

The standard parameterization of the neutrino matrix is carried out using three mixing angles given by

$$
\begin{gathered}
\sin \theta_{12}=\lambda \\
\sin \theta_{23}=B \lambda^{2} \\
\sin \theta_{13}=A \lambda^{3}
\end{gathered}
$$

in which $A, B$ are positive numbers of order unity.

Comparative inspection of the above relationships reveals that the first term in (8) may be associated with the fermion mass hierarchy (11-13), whereas the second term of (8) with the hierarchies present in the mixing matrices (14) and (18)-(19). The key point here is that the numerical value of the Cabibbo angle $\lambda$ falls reasonably close to the reciprocal of the Feigenbaum-like constant for quadratic maps $(\bar{\delta}=4.669 \ldots)$ [1], i.e.

$$
\lambda=O\left(\bar{\delta}^{-1}\right)
$$

We close by noting that these findings are consistent with the following observations:

a) fermion mass and flavor (or weak) eigenstates are mismatched in the SM, as reflected by the separation of terms in (8),

b) masses and mixing matrices are naturally linked to each other in the description of fermion coupling to the Higgs condensate [11]

c) the triplication of SM generations, as well as chiral symmetry breaking of fermions, arise from the continuous approach to chaos of quantum theory near the Fermi scale [13-14].

\section{APPENDIX}

It is worthwhile adding an endnote comment on section 2. The number of working assumptions A1)-A3) can conceivably be reduced to two, namely A1)-A2), on account of the fact that SOC emerges from the complex interaction of a large number of components. As shown in [4], an explicit form of evolution in systems exhibiting complex behavior is provided by the master equation. This equation describes the rate of transition probabilities among various states, which is relevant to non-equilibrium processes such as absorbing phase transitions, directed percolation, and reaction-diffusion [12]. The master equation provides a bridge between SOC and the universal quadratic equation derived from the center manifold theory. Digging deeper into these matters is beyond the scope of this work. 


\section{$\underline{\text { References }}$}

1. https://www.sciencedirect.com/science/article/abs/pii/S1007570406000505

2. Christensen, K. and Moloney, N. R., "Complexity and Criticality", Imperial College Press, 2005.

3. Available at the following sites:

http://www.aracneeditrice.it/aracneweb/index.php/pubblicazione.html?item $=9788854$ $\underline{889972}$

https://www.researchgate.net/publication/278849474 Introduction to Fractional Fi eld Theory consolidated version

4. Nicolis, G., "Physics of far-from-equilibrium systems and self-organization", in "New Physics" pp. 316-347, Ed. Paul Davies, Cambridge Univ. Press, 1992.

5. Shilnikov, L. P. et al., "Methods of Qualitative Theory in Nonlinear Dynamics. Part II", World Scientific series on Nonlinear Science, Series A, Vol. 5, chapter 11, 2001.

6. M. J. Feigenbaum, "Quantitative universality for a class of nonlinear transformations", J. Stat. Phys. 19:25-52 (1978).

7. M. J. Feigenbaum, "The universal metric properties of nonlinear transformations", J. Stat. Phys. 21:669-706 (1979).

8. P. Cvitanovic (ed.), "Universality in Chaos", 2nd Ed. (Adam Bilger, Boston, 1989).

9. P. Collet, J.-P. Eckmann, and H. Koch, "Period Doubling Bifurcations for Families of Maps on Rn”, J. Stat. Phys. 25:1-14 (1981)

10. http://spkuz.narod.ru/2005JSP.pdf

11. https://pdg.lbl.gov/2019/reviews/rpp2019-rev-ckm-matrix.pdf

12. https://arxiv.org/pdf/cond-mat/0001070.pdf

13. Available at the following site:

https://www.researchgate.net/publication/343863324 Addendum to A Bifurcation Model of the Quantum Field

14. Available at the following site:

https://www.researchgate.net/publication/343686626 Three_Generations and Ferm ion Chirality from Universal Bifurcations

15. Available at the following site:

https://www.academia.edu/26552844/An emergence of a quantum world in a se lf organized vacuum a possible scenario 